\title{
The Massive Contact Binary RY Scuti: A WR+OB Progenitor and Its Compact Nebula
}

\author{
Nathan Smith \& Robert D. Gehrz \\ Astronomy Department, University of Minnesota, 116 Church St. SE, \\ Minneapolis, MN 55455, USA
}

\begin{abstract}
RY Scuti is a massive contact binary in a critical Roche-lobe overflow phase, and it may be an immediate progenitor of a $\mathrm{WR}+\mathrm{OB}$ binary system like V444 Cygni. It may also have something in common with the eruptive binary HD 5980 in the SMC. RY Scuti is a nearby eclipsing binary, but basic parameters like the mass and evolutionary state of its individual components seem difficult to nail down because it is a common-envelope system. It is also surrounded by a compact double-ring nebula with geometry and kinematics that have interesting implications for its mass-loss mechanism. Some recent results of highresolution imaging and spectroscopy of the nebula are reviewed here.
\end{abstract}

\section{Introduction}

Soon after massive stars evolve off the main sequence, they presumably take one of two routes to expel their H-rich envelopes before becoming He-burning Wolf-Rayet (WR) stars. A massive single star may enter a transitional phase characterized by heavy mass loss as a Luminous Blue Variable (LBV) or perhaps a very luminous red-supergiant; circumstellar nebulae around these stars have provided valuable clues to their mass-loss histories and evolution. A member of a close binary, on the other hand, may shed its H-rich outer layers through mass loss (or mass transfer) induced by tidal stripping from a companion in a brief Roche-lobe overflow (RLOF) phase. Immediate WR-progenitors like this are rare in the upper HR Diagram, and would surely seem exotic if examined in detail. RY Scuti may be an example of a binary system in just such a pre-WR evolutionary phase, and it is surrounded by a peculiar nebula that reveals clues to its recent mass-loss history and evolutionary state.

\section{RY Scuti: Background}

RY Scuti is a massive (35-90 $\left.\mathrm{M}_{\odot}^{1}\right)$, post-main-sequence eclipsing binary system; one or both of its hot (O6.5 and O9.5) supergiant components has filled its Roche lobe and the system is in a state of overcontact (Milano et al. 1981; Giuricin \&

\footnotetext{
${ }^{1}$ Even though RY Sct is an eclipsing system, there is considerable uncertainty in the total mass, depending on the mass-ratio chosen in fits to the radial velocity and light curves.
} 

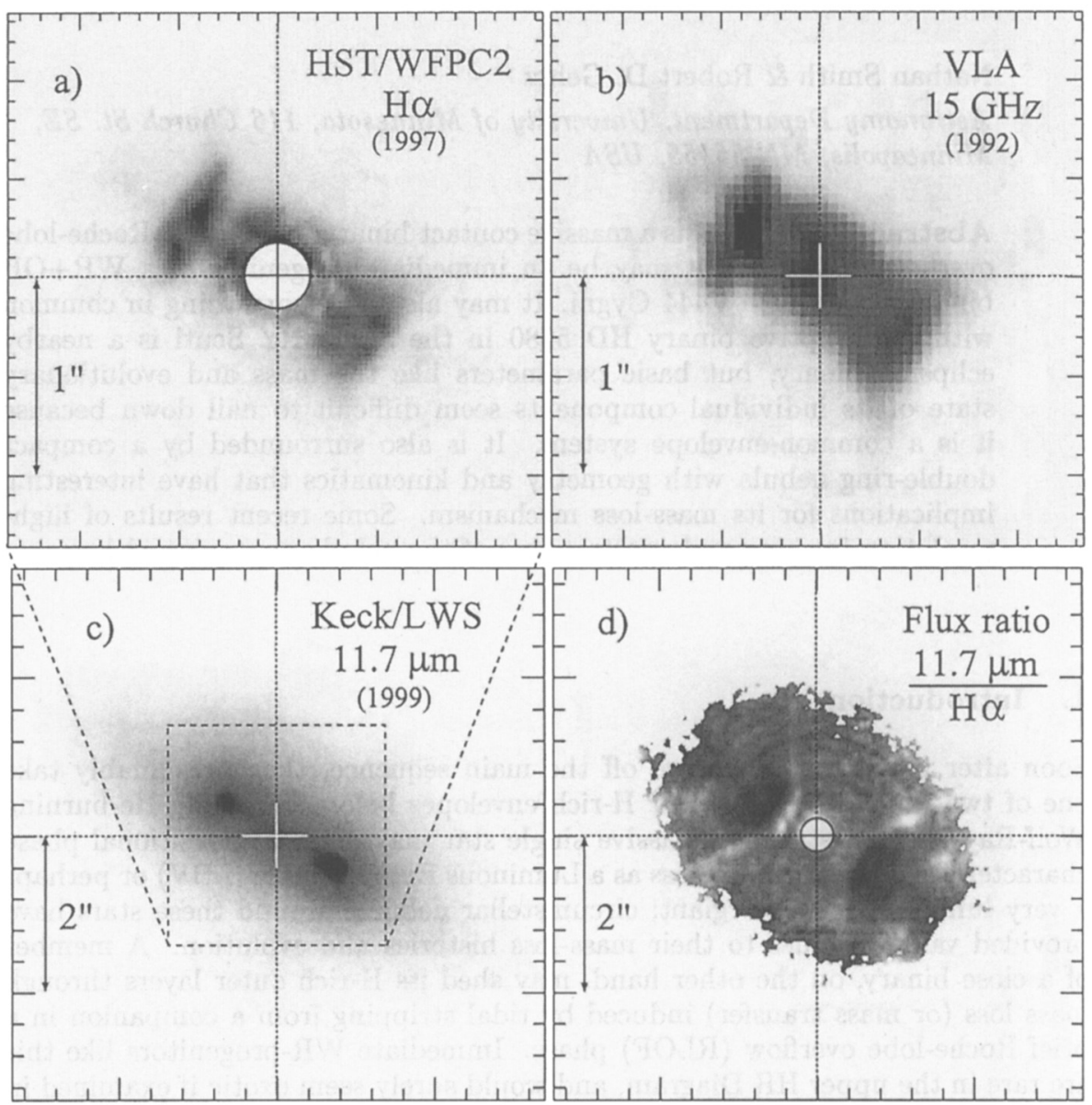

Figure 1. High-resolution images of RY Scuti's nebula. (a) $H S T /$ WFPC2 image in the light of $\mathrm{H} \alpha$ from Smith et al. (1999). (b) Radio free-free continuum image taken with the $V L A$ (See Smith et al. 2001; Gehrz et al. 1995). (c) Thermal-IR dust emission at $11.7 \mu \mathrm{m}$ (Gehrz et al. 2001); note the difference in size scale compared to (a) and (b). (d) Flux-ratio image of panels (a) and (c) showing the relative spatial distribution of dust and ionized gas (dust is shaded dark and hot ionized gas is light in this figure). 


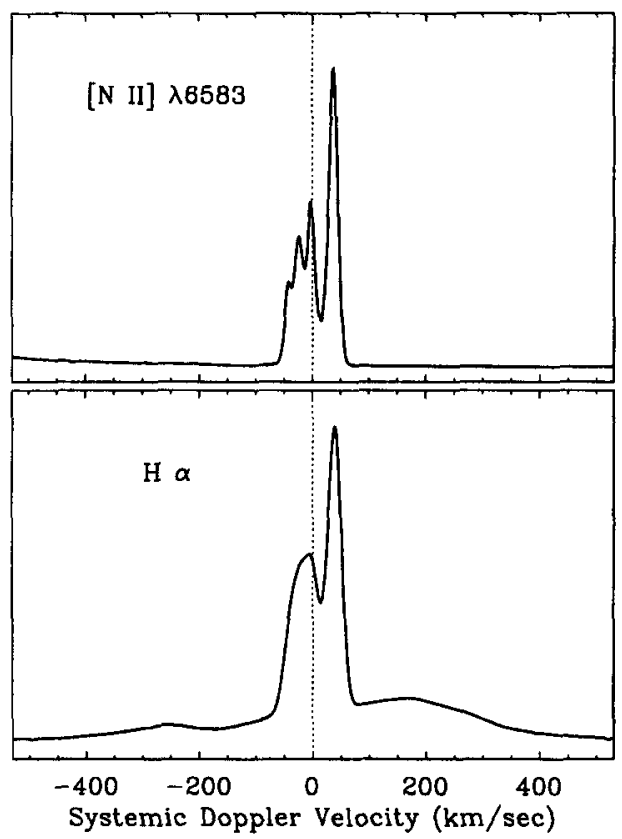

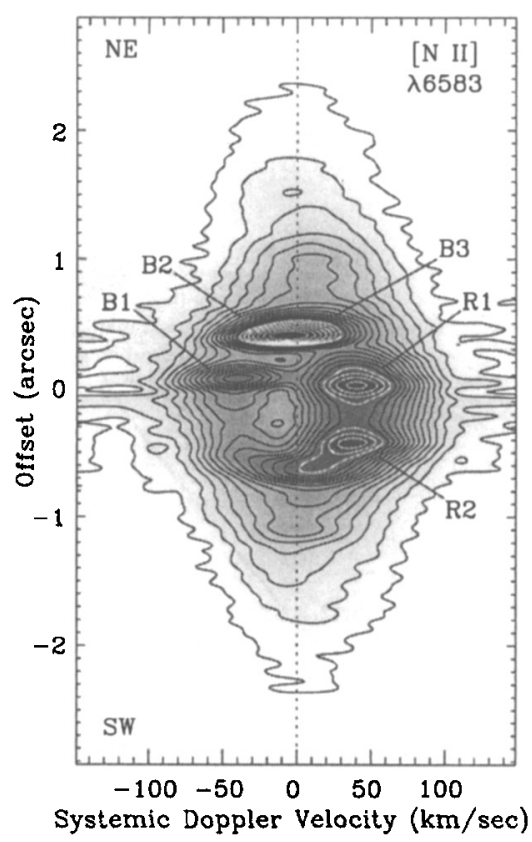

Figure 2. Examples of spectroscopy of RY Scuti (Smith et al. 2002). Left: high-resolution line profiles of [N II] $\lambda 6583$ and $\mathrm{H} \alpha$ as seen in ground-based échelle spectra; the $\mathrm{H} \alpha$ profile also contains a broad component from the stellar wind at $\pm 400 \mathrm{~km} \mathrm{~s}^{-1}$. Right: Long-slit $H S T /$ STIS spectrum of [N II] $\lambda 6583$, with the slit crossing the major axis of the nebula. Clumps in the ring structure corresponding to blueand red-shifted emission peaks are labeled.

Mardirossian 1981; Gehrz et al. 1995). Significant mass-loss and mass-transfer may have already occurred, and the system is probably an immediate progenitor of a WR binary system like V444 Cygni (Antokhina \& Cherapashchuk 1988; Giuricin \& Mardirossian 1981; King \& Jameson 1979).

However, one of the most interesting aspects of RY Scuti is its bright, compact circumstellar nebula, which provides critical clues to mass loss from luminous contact binaries during the RLOF phase. This high-excitation nebula gives rise to a peculiar bright-line optical spectrum, which exhibits prominent [Fe III] lines (Merrill 1928; Edlén \& Swings 1939); historically, RY Scuti is the first object in which these lines were seen and identified.

\section{RY Scuti's Circumstellar Nebula}

\subsection{Imaging}

Since RY Scuti's nebula is relatively compact ( $\sim 1^{\prime \prime}$ across), high spatial resolution is needed to study it. Fig. 1 gives an overview of recent high-resolution 


\section{GEOMETRY OF RY SCUTI'S NEBULA}

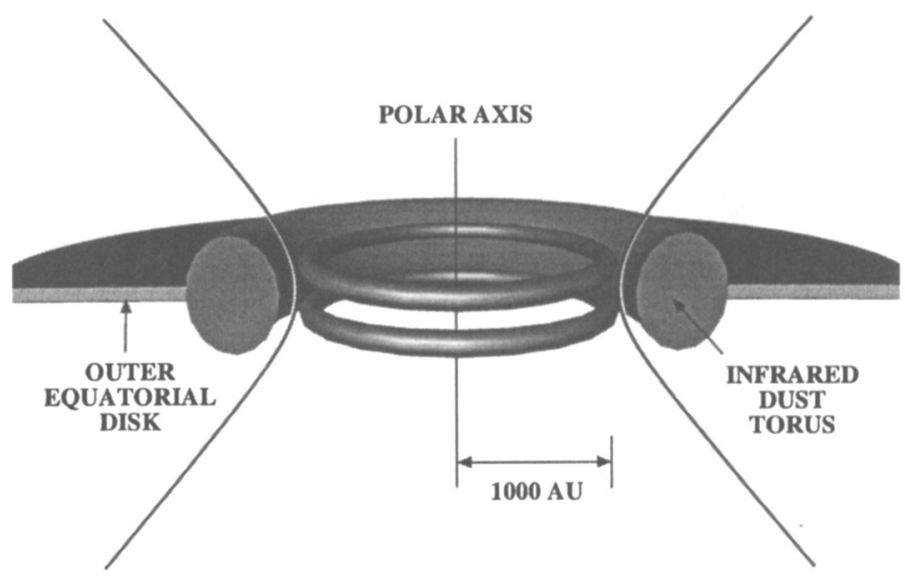

Figure 3. Idealized sketch depicting the geometry of gas and dust in RY Scuti's circumstellar nebula.

imaging of RY Scuti. Ionized gas seen in $\mathrm{H} \alpha$ (Fig. 1a) and radio images (Fig. 1b) shows a peculiar morphology, elongated along P.A. $\approx 58^{\circ}$. The $\mathrm{H} \alpha$ image in particular looks like a pair of ionized rings, parallel to one another, and reminiscent of the rings around SN 1987a, but closer together. Thermal-infrared emission from warm dust (Fig. 1c; Gehrz et al. 2001) is elongated along the same direction and shows a clearly double-peaked morphology. The dust actually resides just outside the ionized gas (Fig. 1d), as if the inner surfaces of the dust clouds are irradiated and ionized by the hot central stars.

\subsection{Spectroscopy}

Every nebular emission line in RY Scuti's spectrum shows essentially the same quadruple-peaked line profile (Fig. 2), except that $\mathrm{H}$ lines are thermally broadened so that the three blueshifted peaks are blended. Emission from the nebula indicates that it is composed of CNO-processed material, and that the total mass of the nebula is roughly $0.003 \mathrm{M}_{\odot}$ (Smith et al. 2002). Spatially-resolved HST/STIS spectra (Fig. 2) with a long-slit aperture crossing the major axis of the nebula show a coherent kinematic structure consistent with an expanding ring rather than bipolar expansion or some other phenomenon. This confirms the impression of a pair of rings given by $\mathrm{H} \alpha$ images mentioned above. Also, the long-slit spectrum in Fig. 2 shows that the quadruple-peaked line profile arises from clumps at various positions around the rings, indicating strong asymmetry in the azimuthal mass distribution, which is surprising in a system with a circularized and tidally-locked orbit.

Broad $\mathrm{H}, \mathrm{He} \mathrm{I}$, and He II lines are also seen in the spectrum, and P Cygni profiles in He I lines indicate a strong stellar wind (Smith et al. 2002). The broad 


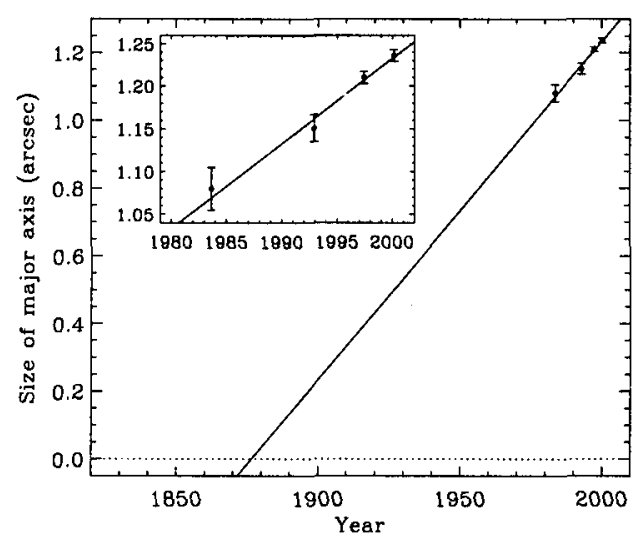

Figure 4. Proper motion measurements of the size across the major axis of RY Scuti's nebula, and a fit indicating a likely ejection date if the expansion has been linear (see Smith et al. 2001).

component of $\mathrm{H} \alpha$ can be seen in Fig. 2 at $\pm 400 \mathrm{~km} \mathrm{~s}^{-1}$, and UV resonance lines indicate faster outflow speeds in the wind (Sahade et al. 1984).

\subsection{Geometry}

Fig. 3 shows a cartoon of the presumed geometry of RY Scuti's nebula inferred from various observational clues. In this context, the double-peaked thermalinfrared emission (Fig. 1c) arises from dust in a limb-brightened, nearly edge-on torus. Ionized gas seen in optical emission lines and radio continuum resides at the inner surface of the dust torus, and appears to spill over the top and bottom of the torus in a bow-shock structure or gas streams (these features are evident in Fig. 1d, but are discussed in more detail by Smith et al. 1999, 2001). Most of the ionized gas at the irradiated inner surfaces of the dust torus makes up a pair of ionized rings, parallel to the equatorial plane. Of course, the hydrodynamic (or magnetohydrodynamic) mechanism that created these rings is still unclear, and worthy of detailed simulations. The nebular mass concentrated in the equatorial plane implies that it was created by mass-loss through the outer Lagrangian point during RLOF, rather than an isotropic wind.

\subsection{Expansion and Age}

The similar morphology in Figs. $1 a$ and $b$ suggests that $\mathrm{H} \alpha$ and radio continuum emission trace the same physical distribution of ionized gas. Two epochs of $H S T$ images in 2000 and 1999 can therefore be combined with earlier VLA images obtained in 1992 and 1983 to investigate the expansion of the nebula (see Smith et al. 2001). Measurements of the size across the major axis are shown in Fig. 4, indicating that RY Scuti's compact nebula is clearly expanding. If the expansion is linear, the motion implies an ejection date in the latter half of the 19th century. At the time of the Henry Draper catalogue, RY Scuti had "dark hydrogen lines," and faint emission lines may have been present. Since at least 
1928 (Merrill 1928), RY Scuti has had a very bright emission line spectrum, and a casual inspection of it would not provoke the comments made in the HD catalogue. It is likely that RY Scuti's spectrum was very different at that time, and it is tempting to attribute that difference to a potential S Doradus-type (or LBV) outburst (see Wolf 1989) that ejected the nebula we see today. The proper motion measurements of RY Scuti's nebula suggest that mass loss during the RLOF phase of very luminous binaries may be characterized by sporadic ejection events, similar to those seen in massive single stars like the LBVs.

We do not know which star in the binary system is responsible for ejecting the nebula. The cooler O9.5 component in RY Scuti resembles the Ofpe/WN9 stars, which are known to be cousins of the LBVs (Stahl et al. 1983). Then again, the famous SMC binary HD 5980 recently suffered a mysterious LBV eruption as well, and the component of that system responsible for the outburst is thought to be a He-enriched O7 supergiant in a pre-WR phase (Koenigsberger et al. 1998; Moffat et al. 1998). Perhaps this gives reason to suspect that the O6.5 supergiant in the RY Scuti system was responsible for ejecting its nebula, instead of the cooler component. In any case, RY Scuti may not be alone among WR-relatives that suffer these outbursts.

Acknowledgments. Support was provided by NASA through STScI, a GSRP fellowship from Goddard Space Flight Center, and the NASA/SIRTF program.

\section{References}

Antokhina, É. A., \& Cherapashchuk, A. M. 1988, Pis'ma Astron. Zh., 14, 252

Edlén, B., \& Swings, J. P. 1939, Observatory, 62, 234

Gehrz, R. D., Smith, N., Jones, B., Puetter, R., \& Yahil, A. 2001, ApJ, 559, 395

Gehrz, R. D. et al. 1995, ApJ, 439, 417

Giuricin, G., \& Mardirossian, F. 1981, A\&A, 101, 138

King, A. R., \& Jameson, R. F. 1979, A\&A, 71, 326

Koenigsberger, G., Auer, L. H., Georgiev, L., \& Guinan, E. 1998, Ap J, 496, 934

Merrill, P. W. 1928, ApJ, 67, 179

Milano, L. et al. 1981, A\&A, 100, 59

Moffat, A. F. J., et al. 1998, ApJ, 497, 896

Sahade, J., Brandi, E., \& Fontenla, J. M. 1984, A\&A SS, 56, 17

Smith, N., Gehrz, R. D., \& Goss, W. M. 2001, AJ, 122, 2700

Smith, N., Gehrz, R. D., Stahl, O., Balick, B., \& Kaufer, A. 2002, ApJ, in press

Smith, N., et al. 1999, AJ, 118, 960

Stahl, O., et al. 1983, A\&A, 127, 49

Wolf, B. 1989, A\&A, 217, 87 